\title{
Tadalafil once daily in the management of erectile dysfunction: patient and partner perspectives
}

\author{
Pierre Costa' \\ Thierry Grivel ${ }^{2}$ \\ Naji Gehchan ${ }^{3}$ \\ 'Service d'Urologie-Andrologie, \\ Hôpital Caremeau, Nîmes, France; \\ ${ }^{2}$ I59, Avenue Sainte-Marguerite, Nice, \\ France; ${ }^{3}$ Eli Lilly and Company, Lilly \\ France - Medical Division, Suresnes, \\ France
}

Correspondence: Pierre Costa Service d'Urologie-Andrologie, Hôpital Caremeau, place du Pr Debré, 30029 Nîmes Cedex 09, France Tel +33 (0)466 683230 Fax +33 (0)4 66683720 Email pierre.costa@chu-nimes.fr

\begin{abstract}
Erectile dysfunction (ED) is a prevalent condition that affects men and their partners. Significant improvements in the sexual lives of these couples have been achieved with the introduction of phosphodiesterase 5 (PDE5) inhibitors. A PDE5 inhibitor is now widely recognized as the first-line therapy for the majority of men with ED. Currently, three PDE5 inhibitors - sildenafil, tadalafil and vardenafil - are approved to be taken as needed in anticipation of sexual activity, but only one of these, tadalafil, has been approved to be taken once daily. The primary aims of this review are to summarize the patients' and partners' viewpoints of ED management with PDE5 inhibitors, and to determine whether once-daily tadalafil can contribute to improving some psychological aspects of ED (such as sexual self-confidence, spontaneity and time concerns) compared with on-demand tadalafil or other PDE5 inhibitors taken by patients with ED.
\end{abstract}

Keywords: erectile dysfunction, once-daily treatment, patient and partner perspectives, phosphodiesterase 5 (PDE5) inhibitor

\section{Introduction}

Male erectile dysfunction (ED) is a prevalent condition defined as the persistent inability to obtain and maintain an erection sufficient to permit satisfactory sexual performance. ${ }^{1,2}$ ED is now recognized as a comorbid condition, especially in men with cardiovascular disease and diabetes mellitus. ${ }^{3}$ Moreover, ED also affects psychological health, eroding self-image, causing emotional distress, impairing marital relations and, therefore, compromising the quality of life of patients and their partners. ${ }^{4,5}$ Before the introduction of oral drugs, pharmacological ED treatment was limited to penile injections or intraurethral insertion of vasoactive agents or prostaglandin E1.

Use of a medication that inhibits phosphodiesterase type 5 (PDE5) is now widely recognized as the first-line therapy for the majority of men with ED. ${ }^{6,7}$ Currently, three PDE5 inhibitors - sildenafil, tadalafil and vardenafil - are approved to be taken as needed before anticipated sexual activity. Many factors can influence patient preference for an ED therapy: drug attributes (efficacy, safety, tolerability and pharmacokinetic properties) as well as behavioral, psychological and relationship factors. Although PDE5 inhibitors are easy to use, men must ingest these oral agents on-demand before sexual activity, and thus remain temporally linked to treatment. For some patients and their partners, planning sexual activity around taking a drug is burdensome. Recent study results demonstrated that chronic administration, with tadalafil ${ }^{8}$ dosed at $2.5,5$ or $10 \mathrm{mg}$ taken once daily, might offer another valuable option for ED treatment., ${ }^{9,10}$ In 2007, 2.5 and $5 \mathrm{mg}$ tadalafil for once-daily use were approved by the European Commission for the treatment of ED, and became available in many states of the European Union. Based on patient choice and physician assessment, tadalafil for once-a-day use is for patients who already respond to PDE5-inhibitor therapy and who anticipate sexual activity at least twice weekly. Once-daily tadalafil represents 
a new therapeutic approach, able to provide some men with uninterrupted treatment for their ED. Therefore, it eliminates the need for planning sex within a limited timeframe and restores or develops freedom and choice of the moment for intercourse as a function of the couple's expectations and/or desires. Using data from the scientific literature and clinical trials, we analyzed patients' and partners' viewpoints of ED management with PDE5 inhibitors, especially once-daily tadalafil. This summary of our findings suggests the interest expressed in an additional therapeutic approach for this indication.

\section{PDE5-inhibitor therapy and ED}

As recommended in the guidelines written by the majority of scientific associations, the first-line therapy for most men with ED is an oral PDE5 inhibitor. These medications are now widely considered safe and effective ED treatments, with reported efficacy rates of $60 \%$ to $70 \% .^{2,6,11}$ Tadalafil, one of the three PDE5 inhibitors currently on the market, is approved in more than 100 countries, including Australia, Brazil, Mexico, Canada, the United States and countries throughout Europe, as an oral treatment for ED. More than 18 million patients worldwide have been treated with tadalafil since its first introduction in February 2003. Like the other PDE5 inhibitors, it was first developed and approved for the treatment of ED on an as-needed basis, with a recommended starting dose of $10 \mathrm{mg}$ in most patients that may be increased to $20 \mathrm{mg}$ or decreased to $5 \mathrm{mg}$, based on individual efficacy and tolerability. PDE5 inhibitors are available by prescription only and are not for everyone. Men should discuss their medical conditions and all medications with their doctors to ensure that such a treatment is right for them and that they are healthy enough for sexual activity.

Until 2007, PDE5 inhibitors were taken on-demand by the majority of patients, being ingested prior to anticipated sexual activity. For the patient, in a way, taking an on-demand therapy assumes that he has a kind of appointment.

Indeed, on-demand PDE5-inhibitor use means making an appointment with oneself or one's partner at a given time during the day or week. Optimization of its on-demand use means that the man identifies the time best adapted to satisfy his desire for sexual intercourse. Of course, he must be assured that his partner is of the same mind. So, the moment of sexual activity is determined by the couple and not the swallowing of the pill. Therefore, good usage of on-demand therapy requires the perfect harmony of the couple, associated with good lines of (non-verbal) communication, and pill intake before the onset of foreplay.
But some patients cannot foresee their desire for sexual intercourse in time for the tablet to be absorbed and become active. They want to have sex at the same time the desire strikes them. ${ }^{12}$ Thus, he should have taken the pill in advance, sometimes with no certainty of having intercourse. Because of its long half-life and 36 to 48 hours of clinical efficacy, tadalafil was preferred by these patients. Some couples, for example, became accustomed to taking tadalafil Friday evening to prepare for the possibility of having intercourse during the weekend. Other patients, and especially their partners, found their sexual desires to be inhibited by the idea of taking a pill. Their solution was to separate, as much as possible, the swallowing of the pill from the onset of sexual activity, so as to 'forget' ingesting the pill. Here again, because of on-demand tadalafil's particularly well-adapted pharmacokinetic properties, especially its 17.5 -hour half-life assuring activity for about 2 days, patients often chose it.

It is certainly this advantage of prolonged activity that explains why preference studies conducted over the past several years found that significantly higher numbers of patients, and their partners, preferred starting or pursuing tadalafil rather than sildenafil or vardenafil. ${ }^{13-17}$

In a double-blind, crossover study, 215 men with ED were randomized to receive 4 weeks of treatment with tadalafil $20 \mathrm{mg}$ or sildenafil $50 \mathrm{mg}$, followed by the alternative treatment after a 1- to 2-week drug washout, to take as needed up to once daily before sexual activity. In order to provide proper dosing instructions for each drug while maintaining investigators and/or patients blinding, two 'nonexistent' placebo arms were added in the protocol, stating that the patients could also be randomized to receive placebo with the tadalafil or sildenafil instruction set. However, it was not a true double-blind study as specific dosing instructions and statements concerning the onset and duration of action of the two PDE5 inhibitors may have introduced a source of bias. In this patient population, with a mean age of 49.8 years, chosen to mimic a new patient consulting his physician for treatment of ED, tadalafil $20 \mathrm{mg}$ was preferred to sildenafil $50 \mathrm{mg}$ (66.3\% vs $33.7 \%$, respectively; $\mathrm{p}<0.001)$ for the initiation of ED therapy. The results of that study also showed that preference did not differ according to patients' age, duration of $\mathrm{ED}$, order in which treatments were received or previous sildenafil exposure. ${ }^{13}$

Another study aimed to determine what percentage of ED patients currently taking sildenafil would switch to tadalafil. Among the 147 patients who were taking sildenafil at stable fixed doses of 25,50 or $100 \mathrm{mg}$ as needed and then received tadalafil $20 \mathrm{mg}, 133(90.5 \%)$ preferred continuing 
oral therapy with tadalafil and $14(9.5 \%)$ with sildenafil $(p<0.001) .{ }^{14}$ To compare preference of sildenafil or tadalafil to treat ED in men naive to PDE5 inhibitors, 367 men with ED were randomized to receive sildenafil for 12 weeks followed by tadalafil for 12 weeks or vice versa. After treatment, 29\% of men chose sildenafil and $71 \%$ chose tadalafil $(\mathrm{p}<0.001)$ for ED therapy during an 8-week extension. ${ }^{15}$

To compare sildenafil, vardenafil and tadalafil, the three PDE5 inhibitors currently available, a prospective, fixed-dose preference study was conducted, with a crossover design. All 90 patients who completed this study were naive to all ED treatments, including PDE5 inhibitors. Drug preference was assessed by evaluating subjective elements that the patients had taken into account in making their choices: 25 (27.8\%) opted to continue sildenafil, 18 (20\%) chose vardenafil and $47(52.2 \%)$ preferred tadalafil. Moreover, although the three medications markedly improved erectile function, statistically significant differences in the International Index of Erectile Function (IIEF) score and responses to the Erectile Dysfunction Inventory of Treatment Satisfaction (EDITS) Questionnaire were found between tadalafil and the two other drugs $(\mathrm{p}<0.0001)$, but not between sildenafil and vardenafil. ${ }^{16}$

All these studies examined several variables, like efficacy, impact on quality of life and self-esteem from a male perspective. But women should also be involved in assessment and treatment decisions when ED is a problem for their partners, and recommendations have been made that research should focus on the impact of ED on women and the couple, rather than simply the man. ${ }^{18}$ In a recent prospective investigation, women were asked for the first time which drug they preferred their partners to use when treated for ED, and why they preferred it. ${ }^{17}$ According to those findings, a large majority of women chose tadalafil over sildenafil (79.2\% and $15.6 \%$, respectively; $\mathrm{p}<0.0001)$. The main reasons the women gave as influencing their treatment preference were more relaxed and natural experiences leading to more intimacy, enjoyment and increased sexual satisfaction, a longer lasting effect of tadalafil, increased sexual frequency and the flexibility offered by the drug in terms of time frames. As noted in that report, the female perspective, compared to that of men, reflected the importance of factors above and beyond obtaining and maintaining an erection for sexual pleasure and enhancement of the couple's relationship.

For the patients, the factors most significantly associated with treatment preference were differences in time concerns, choice of dose, intercourse satisfaction, number of sexual attempts, satisfaction with erection hardness and the absence of or only minor side effects. ${ }^{19}$ Although preference studies have shown that all the PDE5 inhibitors were highly effective, the majority of patients seemed to prefer tadalafil because it is easier to take, can be ingested at any time of the day, and its property of enabling them to achieve an erection, with sexual stimulation, long after taking the drug (eg, 8, 16 or 24 hours later), leading to higher sexual self-confidence and spontaneity and fewer time concerns related to sexual encounters. $^{7,19}$

However, poor treatment satisfaction was reported by some men using on-demand PDE5 inhibitors. They want to be independent of scheduling their love life and to disconnect treatment from sexual intercourse, to enable them and their partners to be free to love and to retrieve a sexual life like before. For these men, the constant association of swallowing a pill and sexual relations, inherent in using on-demand PDE5 inhibitors, could even be a source of psychological and behavioral distress.

As we indicated above, inquiries in large populations of men suffering from ED and treated with PDE5 inhibitors showed that some patients did not respond to on-demand therapies either because they found swallowing the pill just prior to having sex disturbing or they had difficulty identifying when they would have such relations. ${ }^{12}$ Use of on-demand tadalafil, although preferred by them, did not correspond completely to their expectations, because they still had to take a pill in connection with sexual activity. They wanted not to be systematically and continuously obliged to link pill taking with sexual activity.

These observations motivated the development of a new therapeutic approach for this indication, with more frequent or chronic dosing of a PDE5 inhibitor. Therefore, some sexologists, andrologists and urologists devised a new dosing regimen, with tadalafil taken 3 times per week, to determine whether this chronic PDE5-inhibitor dosing regimen might offer a valuable ED-treatment option.

\section{Chronic-dosing regimen: the first step}

The Scheduled Use versus on-demand Regimen Evaluation (SURE) study was the first comparative trial with an alternative dosing schedule. ${ }^{20,21}$ In 4262 men with ED, it investigated treatment efficacy and patient preference for $20 \mathrm{mg}$ tadalafil taken on-demand (maximum one dose per day and before sexual activity) versus 3 times per week for 5 to 6 weeks. After a 1-week washout period, patients were crossed over to the alternate regimen for 5 to 6 weeks. At the end of ondemand and 3 times per week treatment period, a normal EF domain score $(\geq 26$ ) was reported by $60.2 \%$ and $62.3 \%$ 
of patients. Both treatments were equally efficacious and well-tolerated, but a substantial number of men preferred chronic dosing (42.2\%) versus $57.8 \%$ for on-demand. Moreover, patients on the alternative dosing regimen ( 3 times per week) significantly changed their sexual behavior and had sexual attempts distributed over a wide period of time post dosing, with $47 \%$ for on-demand and $71 \%$ for 3 times per week performed beyond the first 4 hours post dosing, preferably in the evening and morning hours. ${ }^{22}$ As the aim of this study was not to demonstrate the superiority of the 3 times per week regimen compared to the on-demand regimen, the results showed that the two regimens provide another treatment option by giving men with ED and their partners unique dosing flexibility with tadalafil. In a retrospective analysis of pooled data from clinical trials of tadalafil using placebocontrolled and open-label study designs, the frequency of serious cardiovascular treatment-emergent adverse events (CVTEAEs) was shown to be low in all groups evaluated. ${ }^{23}$ In addition, the CVTEAE rate in tadalafil-treated patients was independent of the dosing regimen, on-demand or 3 times per week, and comparable with that for placebo-treated patients, thereby demonstrating the safety of the chronicdosing regimen.

Despite the potential advantages of chronic dosing compared to on-demand regimens, taking a treatment 3 times per week seems not to be very easy and convenient for a majority of men with ED. They must note and remember on what days of the week the medication has to be taken. In response to these considerations, a new daily-dosing regimen was investigated, which also allowed a lower PDE5-inhibitor dose to be taken.

\section{IPDE5 and daily dosing: a new treatment option}

Tadalafil differs in several ways from the other two PDE5 inhibitors currently available. Its molecule belongs to a distinct chemical class characterized by a different structure. Because of its $\mathrm{T}_{\max }$ of 2 hours, tadalafil starts to take effect 16 to 60 minutes after intake. Its plasma half-life $\left(\mathrm{T}^{1 / 2}\right)$ is 17.5 hours, as opposed to 4 to 5 hours for sildenafil and vardenafil, which results in improved erectile function and the ability to obtain and maintain one or several erections over the 36 hours following intake. ${ }^{21,25}$ Indeed, clinical trials conducted with orally administered, on-demand tadalafil for patients with ED demonstrated real life improved sexual activity with prolonged windows of opportunity for sexual activity. Furthermore, the rate and extent of tadalafil absorption are not affected by food. ${ }^{24}$
To treat ED, tadalafil should be taken no more than once daily (Cialis ${ }^{\circledR}$, summary of product characteristics). Steadystate plasma concentrations are reached after 5 days of once-daily intake and that regimen affords tadalafil exposure 1.6 times greater than after a single oral dose. ${ }^{26}$ A cumulative plasma exposure equivalent to on-demand doses of $16 \mathrm{mg}$ and $8 \mathrm{mg}$ could be obtained after 5 days of daily ingestion of $10 \mathrm{mg}$ or $5 \mathrm{mg}$ of tadalafil. ${ }^{9}$ Therefore, tadalafil pharmacokinetic characteristics, particularly its long half-life (17.5 hours) and its sustained plasma concentrations after daily dosing, have made it one of the best candidates for once-daily dosing, which may be an attractive, alternative, oral treatment for patients with ED. Numerous clinical trials have shown the efficacy, safety and tolerability of different tadalafil doses taken once daily.

Two open-label studies compared the efficacy and safety of once-daily versus on-demand tadalafil. ${ }^{27,28}$ In the first study, 112 men with moderate-to-severe ED previously unresponsive to on-demand tadalafil were treated with $10 \mathrm{mg}$ tadalafil once daily. ${ }^{27}$ The $10 \mathrm{mg}$ daily dose resulted in mean improvement of IIEF scores of 12.8 and 8.2 compared to pretreatment and on-demand baseline values, respectively ( $p<0.001$ ), and $58 \%$ of intercourse attempts (as assessed with Sexual Encounter Profile diary question 3 [SEP3]) were successfully completed $(\mathrm{p}<0.001)$. More patients reported improved erections with once-daily compared to on-demand tadalafil (69\% versus 42\%, respectively), demonstrating an effective salvage strategy for previous non-responders. The second study evaluated the efficacy, safety and tolerability of on-demand $20 \mathrm{mg}$ versus once-daily $10 \mathrm{mg}$ tadalafil in 145 men with mild to severe ED of various etiologies. ${ }^{28}$ Once-daily tadalafil was associated with a better efficacy profile compared with on-demand tadalafil. All the patients experienced significant improvement in the IIEF-erectile function (EF) domain, but with mean once-daily dose changes significantly higher than for on-demand tadalafil $(p<0.05)$. Moreover, patients taking once-daily tadalafil successfully completed sexual intercourse significantly more frequently than the patients taking tadalafil on-demand $(p<0.05)$. In that study, $72 \%$ of the patients expressed a preference for once-daily dosing. Based on the results of those two open-label studies, the authors concluded that the efficacy of once-daily tadalafil appeared to be better than that of on-demand use.

An initial 12-week, randomized, double-blind and parallel-group study was conducted on 268 men with ED. ${ }^{9}$ The primary outcome end points were changes of IIEF-EF, Sexual Encounter Profile (SEP) question 2 (SEP2; "Where you 
able to insert your penis into partner's vagina?") and SEP3 ("Did your erection last long enough for you to have successful intercourse?") questions. Treatment tolerability and safety were also assessed. For patients treated with placebo or once-daily $5 \mathrm{mg}$ or $10 \mathrm{mg}$ tadalafil, respective score modifications from baseline to end point were: $0.9 \%, 9.7 \%$ and 9.4\% for the IIEF-EF; $11.2 \%, 36.5 \%$ and $39.4 \%$ for SEP2; and of $13.2 \%, 45.5 \%$ and $50.1 \%$ for SEP3. At the end of the study and for the respective treatment groups, $28.3 \%, 84.5 \%$ and $84.6 \%$ of the patients reported improved erections, and $8.3 \%, 51.5 \%$ and $50.5 \%$ reported "no ED" (defined as IIEF 26-30). All comparisons between tadalafil (5 mg or $10 \mathrm{mg}$ ) and placebo were highly significant $(\mathrm{p}<0.001)$. The results also showed that once-daily 5-mg or 10-mg tadalafil was well tolerated. Adverse events described by at least $5 \%$ of the patients were: dyspepsia, headaches, back pain, upper abdominal pain and myalgias. Only nine (3.4\%) patients discontinued therapy because of adverse events.

More recently, the efficacy and safety of lower tadalafil doses taken once daily to treat ED was assessed in a randomized, double-blind, placebo-controlled, paralleldesign study in the US. ${ }^{10}$ Responses to once-daily $2.5 \mathrm{mg}$ or $5 \mathrm{mg}$ tadalafil were compared to placebo, for 24 weeks. The primary efficacy endpoints were change from baseline to 24 weeks in mean IIEF-EF domain score, SEP2 and SEP3. Changes in the Psychological And Interpersonal Relationship Scales (PAIRS) were included in the secondary efficacy endpoints. Efficacy results indicated significantly improved erectile function with tadalafil versus placebo for all three primary study end points. At 24 weeks, more significant mean changes from the baseline IIEF-EF scores were observed for the $2.5 \mathrm{mg}$ (6.1) and $5 \mathrm{mg}$ tadalafil (7.0) groups compared to the placebo group (1.2) ( $\mathrm{p}<0.001)$; with respective baseline-to-end-point changes of $24.3 \%$, $26.2 \%$ and $5.2 \%$ for SEP 2 ; and $31.2 \%, 35.1 \%$ and $9.5 \%$ for SEP3 ( $p<0.001)$. The efficacy results were similar across the 24-week observation period, suggesting no treatment resistance. The efficacy of the $5 \mathrm{mg}$ tadalafil dose was better than that of $2.5 \mathrm{mg}$ tadalafil. This apparent dose-response was observed for IIEF-EF domain scores and responses to SEP2 and SEP3 for patients with mild, moderate or severe ED. At 24 weeks, treatment with tadalafil 2.5 and $5 \mathrm{mg}$ improved PAIRS Sexual Self-confidence Domain scores when compared with placebo (both $\mathrm{p}<0.001$ ), but with no significant differences in the PAIRS Spontaneity Domain scores. Both once-daily tadalafil doses were well tolerated, with low discontinuation rates because of adverse events and a low treatment emergent CVTEAE rate.
Based on the review and evaluation of data from these phase III, randomized, double-blind, placebo-controlled studies, the European Commission approved $2.5 \mathrm{mg}$ and $5 \mathrm{mg}$ tadalafil for once-daily treatment of ED in June 2007. Seven months later, in January 2008, the US Food and Drug Administration approved them. Data from all the clinical trials confirmed that men with ED taking $2.5 \mathrm{mg}$ or $5 \mathrm{mg}$ tadalafil once daily experienced improved erectile function compared with those taking a placebo. When taken without restrictions on the timing of sexual activity, once-daily tadalafil was able to improve erectile function over the course of therapy. Moreover, a recent publication reporting on two open-label extension studies showed that once-daily $5 \mathrm{mg}$ tadalafil for up to 2 years was effective, making it a valid alternative to on-demand tadalafil for men with ED. ${ }^{29}$

Therefore, when tadalafil is taken daily, men can attempt sexual activity at any time. As part of a daily regimen, tadalafil may be taken at $2.5 \mathrm{mg}$ or increased to $5 \mathrm{mg}$, based on individual efficacy and tolerability. This low-dose, oncedaily tadalafil treatment option may be most appropriate for men with ED who anticipate more frequent sexual activity (eg, twice weekly). Furthermore, the couples who like to have choices or want to go back to a certain freedom in their sexual lives will appreciate the availability of a PDE5 inhibitor for once-daily use. For the other men and their partners, treatment with on-demand tadalafil taken will remain an appropriate regimen.

Adverse events reported by the patients taking on-demand tadalafil were mainly headaches, upset stomach, nasal congestion, backache, muscle aches, dizziness and flushing. In clinical trials of once-daily tadalafil, the most commonly reported adverse events were headaches, indigestion, back pain, muscle aches, nasal congestion, flushing and limb pain. Most adverse events reported for on-demand or once-daily tadalafil were transient and generally mild or moderate., ${ }^{9,10}$ Just as on-demand tadalafil has been shown to be safe and well tolerated when taken for up to 2 years, ${ }^{30}$ two dosing studies of long-term, open-label, once-daily tadafil provided evidence that $5 \mathrm{mg}$ tadalafil was also well tolerated for up to 2 years in men with ED. ${ }^{29}$

As for the other PDE5 inhibitors, tadalafil is contraindicated for patients taking nitrates, those with cardiac disease for whom sexual activity is not recommended, and those with non-arteritic anterior ischemic optic neuropathy. Oncedaily tadalafil assures continuous plasma levels, which should be considered when evaluating the potential for interactions with certain medications (eg, nitrates, alphablockers, anti-hypertensives and potent CYP3A4 inhibitors) 
and with substantial amounts of alcohol. PDE5 inhibitors were previously shown to cause no changes in seminal parameters. ${ }^{31}$ A multicenter, randomized, double-blind, placebo-controlled study demonstrated that once-daily $20 \mathrm{mg}$ tadalafil for 9 months (approximately three human spermatogenesis cycles) had no major adverse effects on sperm production or reproductive hormones in healthy men (or those with mild ED) at least 45 years old. ${ }^{32}$ More interestingly, PDE5-inhibitor use by men without ED may lead to a marked reduction of the post-ejaculatory refractory time, indicating that, in normal subjects, PDE5-inhibitor intake may have a positive influence on retumescence following ejaculation in the presence of a continuous erotic stimulation. ${ }^{31,33-35}$

Since once-daily tadalafil $(2.5,5$ or $10 \mathrm{mg})$ was proven effective and well tolerated in the general ED population, a recent study was undertaken to determine the efficacy and tolerability of once-daily $2.5 \mathrm{mg}$ or $5 \mathrm{mg}$ tadalafil by men with ED and diabetes. ${ }^{32}$ This randomized, double-blind, placebo-controlled, multicenter, 12-week study enrolled 298 men with diabetes and ED. Patients who took either tadalafil dose had clinically and significantly improved IIEF-EF and mean success rates for vaginal penetration, completion of intercourse, and overall treatment satisfaction $(\mathrm{p} \leq 0.005$ vs placebo). These results suggest that once-daily $2.5 \mathrm{mg}$ or $5 \mathrm{mg}$ tadalafil may be a good alternative to on-demand therapy for some men with diabetes and ED, eliminating the need to plan sex within a limited time frame.

\section{Conclusion}

The primary purpose of this review was to summarize patient and partner viewpoints on ED management with PDE5 inhibitors, and help determine whether once-daily tadalafil can improve some psychological aspects (such as sexual self-confidence, spontaneity and time concerns) compared to on-demand tadalafil or other PDE5 inhibitors in these patients.

When approved in 2003, on-demand $10 \mathrm{mg}$ or $20 \mathrm{mg}$ tadalafil was the first and only PDE5 inhibitor clinically proven to provide sustained efficacy for up to 36 hours. It transformed the oral treatment of ED by giving men the ability to achieve an erection long after taking the drug. Once-daily $2.5 \mathrm{mg}$ or $5 \mathrm{mg}$ tadalafil provides a new therapeutic alternative for men, who may be looking for a dosing option that avoids scheduling sexual activity, and for couples, who may prefer more convenience and spontaneity in their sexual lives. Taking once daily tadalafil at a lower dose may be as effective as taking higher on-demand doses, and appears to be safe. In agreement with their physicians, patients now have the freedom to choose the dosing regimen most appropriate for them. Some may prefer the up to 36 hours of efficacy provided by on-demand tadalafil while others may want the unique benefit provided by the new once-daily dosing option.

\section{Disclosure}

Dr. Gehchan is employee of Eli Lilly and Company

\section{References}

1. NIH Consensus Conference. Impotence. NIH Consensus Development Panel on Impotence. JAMA. 1993;270:83-90.

2. Wespes E, Amar E, Hatzichristou D, et al. EAU guidelines on erectile dysfunction: an update. Eur Urol. 2006;49:806-815.

3. Hatzichristou D, Gambla M, Rubio-Aurioles E, et al. Efficacy of tadalafil once daily in men with diabetes mellitus and erectile dysfunction. Diabet Med. 2008;25:138-146.

4. Costa P, Grivel T, Giuliano F, et al. Erectile dysfunction: a sentinel symptom? Prog Urol. 2005;15:203-207.

5. Wagner G, Fugl-Meyer KS, Fugl-Meyer AR. Impact of erectile dysfunction on quality of life: patient and partner perspectives. Int J Impot Res. 2000;12(Suppl 4):S144-S146.

6. Cour F, Fabbro-Perray P, Cuzin B, et al. Recommendations to general practice doctors for first line management of erectile dysfunction. Prog Urol. 2005;15:1011-1020.

7. Dean J, Hackett GI, Gentile V, et al. Psychosocial outcomes and drug attributes affecting treatment choice in men receiving sildenafil citrate and tadalafil for the treatment of erectile dysfunction: results of a multicenter, randomized, open-label, crossover study. J Sex Med. 2006;3:650-661.

8. Cialis ${ }^{\circledR}$ (tadalafil). Summary of product characteristics, available at: http://www.fda.gov/cder/foi/label/2007/021368s012lbl.pdf. Accessed September. 2008.

9. Porst H, Giuliano F, Glina S, et al. Evaluation of the efficacy and safety of once-a-day dosing of tadalafil $5 \mathrm{mg}$ and $10 \mathrm{mg}$ in the treatment of erectile dysfunction: results of a multicenter, randomized, double-blind, placebo-controlled trial. Eur Urol. 2006;50:351-359.

10. Rajfer J, Aliotta PJ, Steidle CP, et al. Tadalafil dosed once a day in men with erectile dysfunction: a randomized, double-blind, placebocontrolled study in the US. Int J Impot Res. 2007;19:95-103.

11. Montague DK, Jarow JP, Brodercik GA, et al. Chapter 1: The management of erectile dysfunction: an AUA update. $J$ Urol. 2005;174:230-239.

12. Costa $\mathrm{P}$, Avances C, Wagner L. Erectile dysfunction: knowledge, wishes and attitudes. Results of a French study of 5,099 men aged 17 to 70 . Prog Urol. 2003;13:85-91.

13. Govier F, Potempa AJ, Kaufman J, et al. A multicenter, randomized, double-blind, crossover study of patient preference for tadalafil $20 \mathrm{mg}$ or sildenafil citrate $50 \mathrm{mg}$ during initiation of treatment for erectile dysfunction. Clin Ther. 2003;25:2709-2723.

14. Ströberg P, Murphy A, Costigan T. Switching patients with erectile dysfunction from sildenafil citrate to tadalafil: results of a European multicenter, open-label study of patient preference. Clin Ther. 2003;25:2724-2737.

15. Eardley I, Mirone V, Montorsi F, et al. An open-label, multicentre, randomized, crossover study comparing sildenafil citrate and tadalafil for treating erectile dysfunction in men naïve to phosphodiesterase 5 inhibitor therapy. BJU Int. 2005;96:1323-1332.

16. Tolra JR, Cuadrado Campana JM, Ciuatt LF, et al. Prospective, randomized, open-label, fixed-dose, crossover study to establish preference of patients with erectile dysfunction after taking the three PDE-5 inhibitors. J Sex Med. 2006;3:901-909.

17. Conaglen HM, Conaglen JV. Investigating women's preference for sildenafil or Tadalafil use by their partners with erectile dysfunction: the Partners' Preference Study. J Sex Med. 2008;5:1198-1207. 
18. Colson MH. Women faced with the problem of erectile dysfunction: women's view on a male problem. Prog Urol. 2005;15(4):710-716.

19. Eardley I, Montorsi F, Jackson G, et al. Factors associated with preference for sildenafil citrate and tadalafil for treating erectile dysfunction in men naïve to phosphodiesterase 5 inhibitor therapy: post hoc analysis of data from a multicentre, randomized, open-label, crossover study. BJU Int. 2007;100:122-129.

20. Costa P, Buvat J, Holmes S, et al. Predictors of tadalafil efficacy in men with erectile dysfunction: the SURE study comparing two dosing regimens. $J$ Sex Med. 2006;3(6):1050-1058.

21. Mirone V, Costa P, Damber JE, et al. An evaluation of an alternative dosing regimen with tadalafil, 3 times/week, for men with erectile dysfunction: SURE study in 14 European countries. Eur Urol. 2005;47:846-854.

22. Moncada I, Damber JE, Mirone V, et al. Sexual intercourse attempt patterns with two dosing regimens of tadalafil in men with erectile dysfunction: results from the SURE study in 14 European countries. $J$ Sex Med. 2005;2(5):668-674.

23. Kloner RA, Jackson G, Hutter AM, et al. Cardiovascular safety update of tadalafil: retrospective analysis of data from placebo-controlled and open-label trials of tadalafil with a needed, three times-per-week or once-a-day dosing. Am J Cardiol. 2006;97:1778-1784.

24. Carson CC, Rajfer J, Eardley I, et al. The efficacy and safety of tadalafil: an update. BJU Int. 2004;93:1276-1281.

25. Troconiz IF, Tillmann C, Staab A, et al. Tadalafil population pharmacokinetics in patients with erectile dysfunction. Eur J Clin Pharmacol. 2007;63:583-590.

26. Forgue ST, Patterson BE, Bedding AW, et al. Tadalafil pharmacokinetics in healthy subjects. Br J Clin Pharmacol. 2006;61:280-288.
27. McMahon C. Efficacy and safety of daily tadalafil in men with erectile dysfunction previously unresponsive to on-demand tadalafil. J Sex Med. 2004;1(3):292-300.

28. McMahon C. Comparison of efficacy, safety, and tolerability of on-demand tadalafil and daily dosed tadalafil for the treatment of erectile dysfunction. J Sex Med. 2005;2(3):415-425.

29. Porst H, Rajfer J, Casabé A, et al. Long-term safety and efficacy of tadalafil $5 \mathrm{mg}$ dosed once daily in men with erectile dysfunction. $J \mathrm{Sex}$ Med. 2008;5:2160-2169.

30. Montorsi F, Verheyden B, Meuleman E, et al. Long-term safety and tolerability of tadalafil in the treatment of erectile dysfunction. Eur Urol. 2004;45:339-345.

31. Aversa A, Maazzilli F, Rossi T, et al. Effects of sildenafil (Viagra) administration on seminal parameters and post-ejaculatory refractory time in normal males. Hum Reprod. 2000;15:131-134.

32. Hellstrom WJG, Gittelman M, Jarow J, et al. An evaluation of semen characteristics in men 45 years of age after daily dosing with tadalafil $20 \mathrm{mg}$ : results of a multicenter, randomized, double-blinds, placebocontrolled, 9-month study. Eur Urol. 2008;53:1058-1065.

33. Mondaini N, Ponchietti R, Muir GH, et al. Sildenafil does not improve sexual function in men without erectile dysfunction but does reduce the postorgasmic refractory time. Int J Impot Res. 2003;15:225-228.

34. Ekmekçioğlu O, Inci M, Demirci D, et al. Effects of sildenafil citrate on ejaculation latency, detumescence time, and refractory period: placebocontrolled, double-blind, crossover laboratory setting study. Urology. 2005;65:347-352.

35. Wang WF, Minhas S, Ralph DJ. Phosphodiesterase 5 inhibitors in the treatment of premature ejaculation. Int J Androl. 2006;29:503-509. 
